\title{
Lovastatin, not Simvastatin, Corrects Core Phenotypes in the Fragile X Mouse Model
}

\author{
Melania Muscas, ${ }^{1}$ Susana R. Louros, ${ }^{1}$ and ${ }^{1}$ Emily K. Osterweil ${ }^{1}$
}

https://doi.org/10.1523/ENEURO.0097-19.2019

${ }^{1}$ Centre for Discovery Brain Sciences, Simons Initiative for the Developing Brain, University of Edinburgh, Edinburgh EH8 9XD, United Kingdom

\section{Visual Abstract}

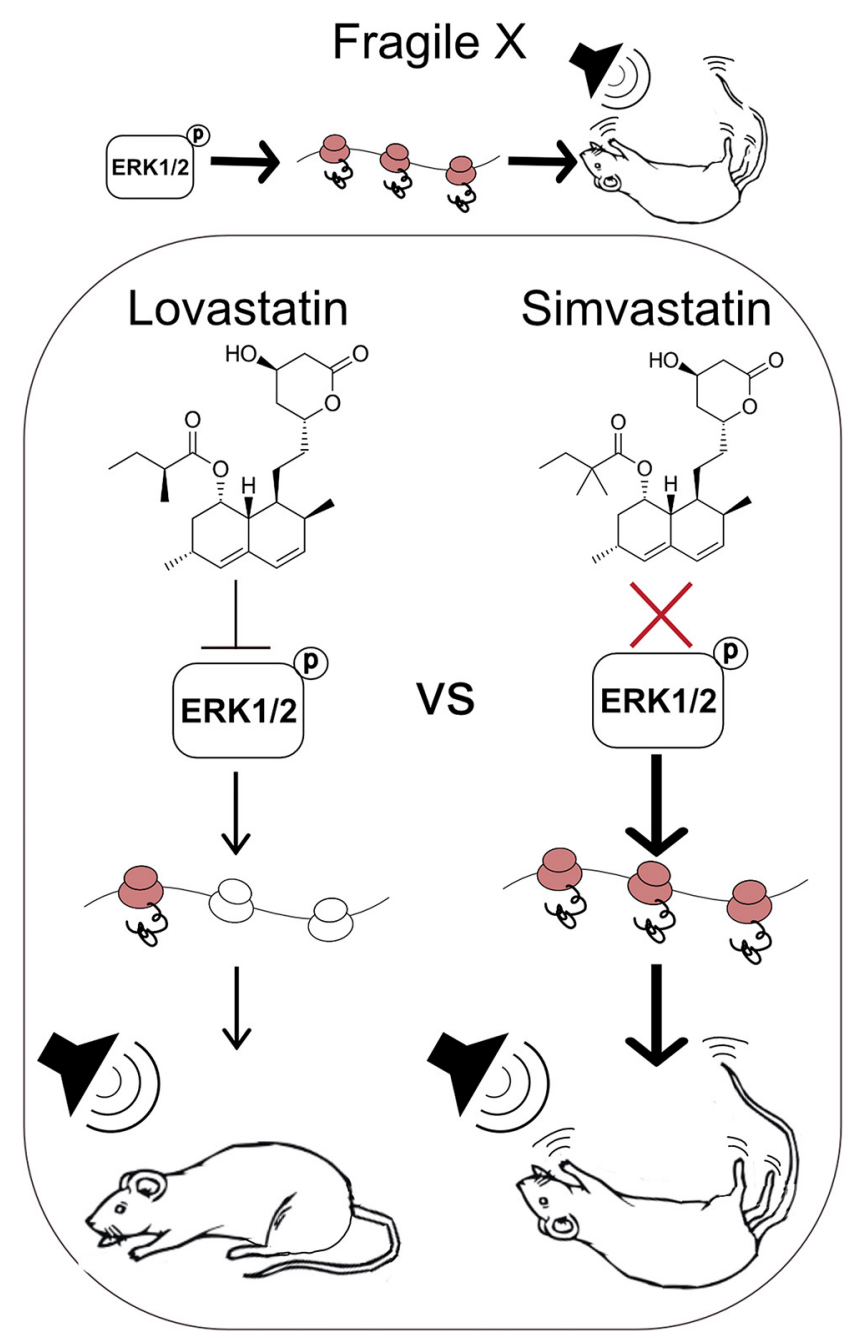

The cholesterol-lowering drug lovastatin corrects neurological phenotypes in animal models of fragile $X$ syndrome (FX), a commonly identified genetic cause of autism and intellectual disability (ID). The therapeutic efficacy of lovastatin is being tested in clinical trials for FX; however, the structurally similar drug simvastatin has been proposed as an alternative due to an increased potency and brain penetrance. Here, we perform a side-by-side comparison of the effects of lovastatin and simvastatin treatment on two core phenotypes in Fmr1 ${ }^{-/ y}$ mice versus 
WT littermates: excessive hippocampal protein synthesis and susceptibility to audiogenic seizures (AGSs). We find that simvastatin does not correct excessive hippocampal protein synthesis in the $F m r 1^{-1 / y}$ hippocampus at any dose tested. In fact, simvastatin significantly increases protein synthesis in both $F m r 1^{-1 y}$ and WT. Moreover, injection of simvastatin does not reduce AGS in the $F m r^{-1 y}$ mouse, while lovastatin significantly reduces AGS incidence and severity versus vehicle-treated animals. These results show that unlike lovastatin, simvastatin does not correct core phenotypes in the $F \mathrm{mr}^{-/ y}$ mouse model.

Key words: ERK; FMR1; fragile X; lovastatin

\section{Significance Statement}

The statin drug lovastatin is in clinical trials for the treatment of fragile X syndrome ( $F X)$, and the structurally similar drug simvastatin has been proposed as a viable alternative. This study compares the efficacy of these drugs for ameliorating two major phenotypes in the FX mouse model and shows that although lovastatin is effective in correcting excessive protein synthesis and audiogenic seizures (AGSs), simvastatin fails to correct either phenotype. These results suggest caution should be used when assuming simvastatin is a suitable substitute for lovastatin with respect to the treatment of FX or other neurodevelopmental disorders.

\section{Introduction}

Fragile $\mathrm{X}$ syndrome $(\mathrm{FX})$ is a monogenic neurodevelopmental disorder characterized by severe intellectual disability (ID), autism, hypersensitivity to sensory stimulation and epilepsy (Lozano et al., 2014). FX occurs in 1:4000 males and 1:8000 females, making it one of the most commonly identified genetic causes of autism and ID (Hagerman et al., 2009; Lozano et al., 2014). The FMR1 gene mutated in FX encodes fragile $X$ mental retardation protein (FMRP), which represses mRNA translation in neurons (Ashley et al., 1993; Darnell et al., 2011). Studies of the $F m r 1^{-1 y}$ mouse model of $\mathrm{FX}$ reveal that excessive cerebral protein synthesis is a major consequence of Fmr1 deletion (Qin et al., 2005; Dölen et al., 2007; BerryKravis et al., 2017; Stoppel et al., 2017b), which can be normalized through antagonism of metabotropic glutamate receptor $5\left(\mathrm{mGlu}_{5}\right)$ or the downstream extracellular regulated kinase 1/2 (ERK1/2) MAP kinase and mammalian target of rapamycin (mTOR)-p70 S6 kinase (p70S6K) signaling pathways (Dölen et al., 2007; Osterweil et al., 2010; Sharma et al., 2010; Michalon et al., 2012; Wang

Received March 15, 2019; accepted May 13, 2019; First published May 30, 2019.

The authors declare no competing financial interests.

Author contributions: M.M., S.R.L., and E.K.O. designed research; M.M. and S.R.L. performed research; M.M. and S.R.L. analyzed data; M.M., S.R.L., and E.K.O. wrote the paper.

This work was supported by the Wellcome Trust/Royal Society Sir Henry Dale Fellowship 104116/Z/14/Z, the Medical Research Council Grant MR/ M006336/1, and Simons Initiative for the Developing Brain.

Acknowledgements: We thank the contributions of all members of the Osterweil lab, with special thanks to Sophie Thomson, Sang Seo, Steph Barnes, and Caoimhe Kirby. We also thank Peter Kind and Mike Cousin for helpful insights and advice.

Correspondence should be addressed to Emily K. Osterweil at emily.osterweil@ed.ac.uk

https://doi.org/10.1523/ENEURO.0097-19.2019

Copyright (C) 2019 Muscas et al.

This is an open-access article distributed under the terms of the Creative Commons Attribution 4.0 International license, which permits unrestricted use, distribution and reproduction in any medium provided that the original work is properly attributed. et al., 2012). These strategies correct multiple neurologic phenotypes in the $\mathrm{Fmr}^{-/ y}$ mouse, including an enhanced susceptibility to audiogenic seizures (AGSs; Bear et al., 2004; Dölen et al., 2007; Osterweil et al., 2010; Stoppel et al., 2017a). The current challenge is to successfully transition these therapeutic approaches to the clinic.

Previous work shows that the statin drug lovastatin, currently used for the treatment of high cholesterol in adults and children, resolves neuropathology in the $F m r 1^{-1 y}$ mouse model (Osterweil et al., 2013). Lovastatin normalizes protein synthesis by reducing the farnesylation and subsequent activation of the GTPase Ras, which lies upstream of the ERK1/2 signaling pathway (Schafer et al., 1989; Mendola and Backer, 1990). By this mechanism, lovastatin has also been shown to successfully correct electrophysiological and behavioral phenotypes in the mouse model of neurofibromatosis type 1 (NF1), a neurodevelopmental disorder of excess Ras (Li et al., 2005). In contrast to ERK1/2, the mTOR-p70S6K pathway activated by the GTPase Rheb is not altered by lovastatin suggesting the impact on farnesylation does not extend to all targets (Osterweil et al., 2013).

In the $\mathrm{Fmr1}^{-/ y}$ mouse, the reduction of Ras-ERK1/2 by lovastatin ameliorates hippocampal epileptogenesis and neocortical hyperexcitability and significantly reduces the incidence of AGS (Osterweil et al., 2013). The AGS phenotype is one of the most robust behavioral phenotypes seen in the $F \mathrm{Fr}^{-1 / y}$ mouse, and it models the epilepsy observed in FX patients (Musumeci et al., 2000; BerryKravis, 2002). Several previous studies have used AGS as a benchmark for determining the efficacy of potential treatment strategies, consistently finding a positive correlation between treatment efficacy at reducing seizure incidence and correction of other pathologies (Yan et al., 2005; Dölen et al., 2007; Osterweil et al., 2010, 2013; Busquets-Garcia et al., 2013; King and Jope, 2013). Based on the positive outcome with lovastatin in $\mathrm{Fmr1}^{-1 / y}$ animal models, two open-label clinical trials tested the viability of lovastatin for the treatment of FX (Çaku et al., 2014; Pellerin et al., 2016). Both studies revealed a sig- 
nificant improvement with lovastatin treatment, and a double-blind placebo-controlled trial is ongoing (BerryKravis et al., 2017).

Interestingly, the availability of lovastatin is not widespread in Europe and is not licensed for use in the United Kingdom. Instead, the drug simvastatin has been proposed as an alternative therapeutic. Simvastatin is a structurally similar derivative of lovastatin that is twice as potent, with a daily dose of only $10 \mathrm{mg}$ reducing cholesterol by 25-30\% compared to $20 \mathrm{mg}$ of lovastatin (Jones et al., 1998; Schaefer et al., 2004; Neuvonen et al., 2008). Simvastatin is also more brain penetrant than lovastatin, suggesting it may be a better option for neurologic indications (Tsuji et al., 1993). However, simvastatin has not been investigated in the $F m r 1^{-/ y}$ model, and the impact on Ras-ERK1/2 signaling in the brain is not well established. This information is critical, as clinical trials in NF1 have recently shown that lovastatin has a beneficial impact on cognitive function whereas simvastatin does not (Krab et al., 2008; Alabama-Birmingham and NCI, 2009, 2010; van der Vaart et al., 2013; Bearden et al., 2016; Payne et al., 2016).

In this study, we performed a side-by-side comparison of lovastatin and simvastatin to answer the simple but important question of whether there is a similar rescue of pathology in the $F m r 1^{-/ y}$ mouse. We focused on two core phenotypes in the $F m r 1^{-1 y}$ model: excessive protein synthesis and enhanced susceptibility to AGS. Importantly, our results clearly show that lovastatin, but not simvastatin, is effective in reducing ERK1/2 activity and normalizing protein synthesis in the $F m r 1^{-/ y}$ hippocampus. This suggests that simvastatin acts via a different mechanism from lovastatin with respect to ERK1/2-driven protein synthesis in the brain. To examine whether there was a similar impact on pathology, we performed a thorough AGS analysis using multiple doses of simvastatin. The results of these experiments show that simvastatin does not reduce the incidence or severity of AGS in the $F m r 1^{-/ y}$ mouse under conditions where lovastatin is significantly effective. Together, this evidence suggests simvastatin may not be a suitable replacement for lovastatin with respect to the treatment of $\mathrm{FX}$.

\section{Materials and Methods}

\section{Mice}

All mice tested were male and were naive to drug and behavioral testing before experimentation. Mice were group housed with unrestricted food and water access and a 12/12 $\mathrm{h}$ light/dark cycle. Room temperature was maintained at 21 $\pm 2^{\circ} \mathrm{C}$. All animal procedures were performed in accordance with the University of Edinburgh animal care committee's regulations and the United Kingdom Animals Act. Fmr1'-ly mice (The Jackson Laboratory 003025, RRID:IMSR_JAX: 003025) were maintained on either a C57BL/6J (Charles River) or a mixed C57BL/6J x FVB background (C57BL/6J backcrossed to FVB by two generations).

\section{Metabolic labeling}

Hippocampal slices were prepared from male littermate wild-type (WT) and $F m r 1^{-/ y}(\mathrm{KO})$ C57BL/6J mice [postnatal day (P)25-P32], in an interleaved fashion, with the experimenter blind to genotype. Mice were anaesthetized with isoflurane, and the hippocampus was rapidly dissected in ice-cold ACSF (124 mM NaCl, $3 \mathrm{mM} \mathrm{KCl,} 1.25 \mathrm{mM}$ $\mathrm{NaH}_{2} \mathrm{PO}_{4}, 26 \mathrm{mM} \mathrm{NaHCO}, 10 \mathrm{mM}$ dextrose, $1 \mathrm{mM}$ $\mathrm{MgCl}_{2}$, and $2 \mathrm{mM} \mathrm{CaCl}_{2}$, saturated with $95 \% \mathrm{O}_{2}$ and $5 \%$ $\mathrm{CO}_{2}$ ). Slices (500 $\mu \mathrm{m}$ thick) were prepared using a Stoelting Tissue Slicer and transferred into $32.5^{\circ} \mathrm{C}$ ACSF (saturated with $95 \% \mathrm{O}_{2}$ and $5 \% \mathrm{CO}_{2}$ ) within $5 \mathrm{~min}$. Slices were incubated in $32.5^{\circ} \mathrm{C} \mathrm{ACSF}$ for $4 \mathrm{~h}$ to allow for recovery of protein synthesis then transferred to ACSF containing 25 $\mu \mathrm{M}$ Actinomycin $\mathrm{D}$ (Tocris) plus either vehicle $(0.05 \%$ DMSO in $\mathrm{ddH}_{2} \mathrm{O}$ ), $50 \mu \mathrm{M}$ lovastatin active form (CAS 75225-50-2; Calbiochem Merck Millipore), or 0.1-5 $\mu \mathrm{M}$ simvastatin active form (CAS 101314-97-0; Cayman Chemical) for $30 \mathrm{~min}$. To measure new protein synthesis, slices were then transferred to fresh ACSF with $10 \mu \mathrm{Ci} / \mathrm{ml}$ ${ }^{35}$ S-Met/Cys (PerkinElmer) containing vehicle (veh) or drug for another $30 \mathrm{~min}$.

After labeling, slices were homogenized in ice-cold buffer (10 mM HEPES, pH 7.4, 2 mM EDTA, 2 mM EGTA, 1\% Triton $\mathrm{X}-100$, protease inhibitors, and phosphatase inhibitors) and incubated in trichloroacetic acid (TCA; 10\% final) for $10 \mathrm{~min}$ on ice before being centrifuged at 16,000 rpm for $10 \mathrm{~min}$. The pellet was washed in ice-cold $\mathrm{ddH}_{2} \mathrm{O}$ and resuspended in $1 \mathrm{~N} \mathrm{NaOH}$ until dissolved, and the $\mathrm{pH}$ was readjusted to neutral using $0.33 \mathrm{~N} \mathrm{HCl}$. Triplicates of each sample were subjected to scintillation counting and protein concentration assay kit (Bio-Rad). Counts per minute (CPM) were divided by protein concentration, and this was normalized to the CPM from the ACSF used for incubation. For display purposes, example slice homogenates were resolved on SDS-PAGE gels, transferred to nitrocellulose and exposed to a phosphorimaging screen (GE Healthcare). Phosphorimages were acquired using a Typhoon scanner (GE Healthcare) and compared to total protein staining of the same membrane.

\section{Immunoblotting}

Samples were loaded on SDS-PAGE gels, with all conditions per littermate pair (i.e., WT veh, KO veh, WT drug, $\mathrm{KO}$ drug) present on the same gel (Extended Data Fig. 2-1). Samples were coded such that the experimenter was blinded to genotype and treatment. Gels were transferred to nitrocellulose and stained for total protein with the Memcode Reversible staining kit (Pierce). To immunoblot for ERK1/2 and p70S6K in the same samples, membranes were cut at 75,50 , and $37 \mathrm{kDa}$ as shown in Extended Data Figure 2-1. For membranes probed for $\mathrm{p}$-p70S6K, the portion of membrane above $75 \mathrm{kDa}$ was removed to eliminate the background p85S6K band recognized by this antibody. Each membrane was then blocked with $5 \%$ BSA in TBS $+0.1 \%$ Tween 20 and incubated in primary antibody overnight at $4^{\circ} \mathrm{C}$ [Cell Signaling Technology; phospho-ERK1/2 (Thr202/Tyr204) 1:2000 (\#9106, RRID:AB_331768), ERK1/2 1:2000 (\#9102, RRID:AB_330744), phospho-p70S6K (Thr389) 1:1000 (\#9234, RRID:AB_2269803), p70S6K 1:1000 (\#2708, RRID:AB_390722); Extended Data Fig. 2-1]. Membranes were then washed, incubated with HRP-conjugated sec- 
ondary antibodies for 30 min (Cell Signaling; RRID: AB_330924 and RRID:AB_2099233), and developed with Clarity ECL (Bio-Rad). Densitometry was performed on scanned blot films using Image Studio Lite software, RRID: SCR_013715.

To compare phopho to total for each target in the same lane, membranes developed for phospho [i.e., phosphorylated (p-)ERK1/2] were stripped and reprobed for total (i.e., ERK1/2). Phosphorylation of target proteins was calculated as a ratio of phospho to total. To correct for blot-to-blot variance, each signal was normalized to the average signal of all lanes on the same blot. Values are shown as a percentage of average WT vehicle for graphical purposes. All membranes were analyzed with experimenter blind to genotype and treatment.

\section{AGSs}

Test cohorts were counterbalanced for genotype and treatment. Naive WT and Fmr1 ${ }^{-1 y}$ male P18-P29 mice bred on a mixed C57BL/6J x FVB background were weighed and injected intraperitoneally with $3 \mathrm{mg} / \mathrm{kg} \mathrm{sim-}$ vastatin prodrug (CAS 79902-63-9), $50 \mathrm{mg} / \mathrm{kg}$ simvastatin active form (CAS 101314-97-0), or $100 \mathrm{mg} / \mathrm{kg}$ lovastatin active form (CAS 75225-50-2) or respective vehicle (3\%, $20 \%$, or $50 \%$ DMSO + $10 \%$ Tween 80 in PBS). Animals were then transferred to a quiet $(<60-\mathrm{dB}$ ambient sound) room for $1 \mathrm{~h}$. For testing, animals were moved to a transparent test chamber equipped with speakers and a webcam and allowed to habituate for $1 \mathrm{~min}$. Audiogenic stimulation (recorded sampling of a modified personal alarm) was passed through an amplifier and $2 \times 50-\mathrm{W}$ speakers (KRK Rokit RP5 G3 Active Studio Monitor) to produce a stimulus of $>130 \mathrm{~dB}$ for 2 min. A decibel meter was placed at a standard distance from the speakers to ensure a stable emission of sound throughout each session. Incidence and severity of seizures was scored and video files for each session were saved. Latency was measured as the number of seconds between onset of the AGS stimulus and appearance of the first seizure. Stages of AGS severity were assigned according to previous work as follows: (1) wild running (WR; pronounced, undirected running and thrashing), (2) clonic seizure (violent spasms accompanied by loss of balance), or (3) tonic seizure (loss of movement and postural rigidity in limbs and tail). Any animal that reached tonic seizure was immediately humanely killed. All injections, testing and scoring was performed with the experimenter blind to genotype and treatment.

\section{Statistics}

Statistical testing was performed using GraphPad Prism 6 software, RRID: SCR_002798. For biochemistry experiments, outliers $>2$ SD from the mean were removed and significance determined by repeated measures twoway ANOVA and post hoc Sidak's multiple comparisons test. Significance for AGS incidence was determined using Fisher's exact test. AGS severity score distributions were tested for normality and found to be non-normal by Shapiro-Wilk test. These score distributions were then statistically compared using a Mann-Whitney $U$ test for analysis of ordinal datasets with non-normal distributions.
Significant differences in latency to first seizure were determined using unpaired two-tailed Student's $t$ test. Results of all statistical analyses are reported in detail in the statistical table (Table 1) and figure legends.

\section{Results}

\section{Lovastatin, but not simvastatin, normalizes excessive protein synthesis in the Fmr1 ${ }^{-/ y}$ hippocampus}

Previous work shows that lovastatin normalizes excessive protein synthesis in the $F \mathrm{Fr}^{-/ y}$ hippocampus through reduction of Ras-ERK1/2 activation, which corrects epileptogenic phenotypes (Osterweil et al., 2013). To examine whether the same effect is seen with simvastatin, we used a metabolic labeling assay in hippocampal slices designed to assess protein synthesis in an intact preparation under physiologic conditions. Hippocampal slices were prepared from juvenile WT and $\mathrm{Fmr1}^{-1 /}$ littermates, blind to genotype, and allowed to recover in oxygenating ACSF. Following this, slices were preincubated with Actinomycin $D$ to block transcription, and new protein synthesis was labeled through incorporation of ${ }^{35} \mathrm{~S}$-labeled methionine/cysteine mix (Fig. 1A).

Previous experiments tested a range of $10-50 \mu \mathrm{M}$ lovastatin and showed that $50 \mu \mathrm{M}$ was effective in normalizing protein synthesis in the $F m r 1^{-1 y}$ hippocampus (Osterweil et al., 2013). To ensure that we could recapitulate these results, we measured protein synthesis in WT and $F m r 1^{-1 y}$ slices $\pm 50 \mu \mathrm{M}$ lovastatin (Fig. 1B). As expected, our experiments revealed a significant correction of excessive protein synthesis with lovastatin in the $\mathrm{Fmr1}^{-1 / y}$ mouse (WT veh $=100 \pm 1.48 \%$, WT lova $=$ $100.06 \pm 4.87 \%, \mathrm{KO}$ veh $=117.97 \pm 4.27 \%, \mathrm{KO}$ lova $=$ $106.04 \pm 4.93 \%$; WT vs $\mathrm{KO}$ veh $p=0.0032$, KO veh vs lova $p=0.0368 ; n=12$ ). Next, we tested the efficacy of simvastatin using the same assay system. As simvastatin is twice as potent as lovastatin with respect to reducing plasma LDL cholesterol levels in patients, we tested a lower dose range of simvastatin in our metabolic labeling assay (Tsuji et al., 1993; Jones et al., 1998; Schaefer et al., 2004; Neuvonen et al., 2008). This concentration is consistent with previous studies of simvastatin in cultured neurons (Lim et al., 2006; Johnson-Anuna et al., 2007; Mans et al., 2010). Interestingly, we find that simvastatin treatment not only fails to reduce protein synthesis in the $F m r 1^{-1 y}$ hippocampus, it causes a significant increase in both WT and $F m r 1^{-1 /}$ slices at $5 \mu \mathrm{M}$ (WT vehicle $=100 \pm$ $2.70 \%$, WT $5 \mu \mathrm{M}=153.5 \pm 6.32 \%, \mathrm{KO}$ vehicle $=111 \pm$ $4.27 \%, \mathrm{KO} 5 \mu \mathrm{M}=170.60 \pm 9.43 \%$; WT veh vs $5 \mu \mathrm{M} \mathrm{p}$ $=0.0001, \mathrm{KO}$ veh vs $5 \mu \mathrm{M} p<0.0001 ; n=10$; Fig. $1 C$ ).

This puzzling increase in protein synthesis led us to wonder whether a reduced concentration of simvastatin might be more appropriate. To test this, we exposed slices to vehicle or simvastatin at concentrations of 0.1 $0.5 \mu \mathrm{M}$. Surprisingly, we find that even at these lower concentrations simvastatin causes a dose-dependent increase in protein synthesis, worsening the $\mathrm{Fmr}^{-1 / y}$ phenotype $(\mathrm{WT}$ veh $=100 \pm 2.21 \%, \mathrm{WT} 0.1 \mu \mathrm{M}=106.99 \pm$ $3.51 \%$, WT $0.3 \mu \mathrm{M}=117.79 \pm 4.08 \%$, WT $0.5 \mu \mathrm{M}=$ $124.13 \pm 4.23 \%, \mathrm{KO}$ veh $=115.61 \pm 3.48 \%, \mathrm{KO} 0.1 \mu \mathrm{M}$ 
Table 1. Statistics table

\begin{tabular}{|c|c|c|c|c|}
\hline Figure & Data structure & Statistical test & Sample size & Statistical data \\
\hline \multicolumn{5}{|c|}{ Figure $1 B$, metabolic labelling of protein synthesis with $50 \mu \mathrm{M}$ lovastatin/vehicle } \\
\hline & Normally distributed & Two-way RM ANOVA & $\begin{array}{l}N=12 \\
\text { per group }\end{array}$ & Genotype: $p=0.0106$ \\
\hline $\begin{array}{l}\text { WT veh vs Fmr1 } \\
\text { KO veh }\end{array}$ & Normally distributed & Sidak's post hoc & $\begin{array}{l}N=12 \\
\text { per group }\end{array}$ & $\begin{array}{l}\text { Cl: }-0.2916 \text { to }-0.06786 \text {, } \\
p=0.0032\end{array}$ \\
\hline $\begin{array}{l}\text { WT } 50 \mu \mathrm{M} \text { lovastatin vs } \\
\text { Fmr1 KO } 50 \mu \mathrm{M} \\
\text { lovastatin }\end{array}$ & Normally distributed & Sidak's post hoc & $\begin{array}{l}N=12 \\
\text { per group }\end{array}$ & $\begin{array}{l}\mathrm{Cl}:-0.1716 \text { to } 0.05214 \\
p=0.3516\end{array}$ \\
\hline $\begin{array}{l}\text { Fmr1 KO vehicle vs } \\
\text { Fmr1 KO } 50 \mu \mathrm{M} \\
\text { lovastatin }\end{array}$ & Normally distributed & Sidak's post hoc & $\begin{array}{l}N=12 \\
\text { per group }\end{array}$ & $\begin{array}{l}\text { Cl: } 0.007476 \text { to } 0.2312 \\
p=0.0368\end{array}$ \\
\hline \multicolumn{5}{|c|}{ Figure $1 C$, metabolic labelling of protein synthesis with 1-5 $\mu \mathrm{M}$ simvastatin/vehicle } \\
\hline & Normally distributed & Two-way RM ANOVA & $\begin{array}{l}N=10 \\
\text { per group }\end{array}$ & $\begin{array}{l}\text { Treatment: } \\
p<0.0001 \\
\text { genotype: } \\
p=0.0294\end{array}$ \\
\hline WT veh vs KO veh & Normally distributed & Sidak's post hoc & $\begin{array}{l}N=10 \\
\text { per group }\end{array}$ & $\begin{array}{l}\mathrm{Cl}:-0.3188 \text { to } 0.09835 \\
p=0.3451\end{array}$ \\
\hline WT veh vs KO veh & Normally distributed & Paired $t$ test & $\begin{array}{l}N=10 \\
\text { per group }\end{array}$ & $\begin{array}{l}\mathrm{Cl}: 0.008558 \text { to } 0.2119 \\
p=0.0366\end{array}$ \\
\hline $\begin{array}{l}\text { WT veh vs WT } 5 \mu \mathrm{M} \\
\text { simvastatin }\end{array}$ & Normally distributed & Sidak's post hoc & $\begin{array}{l}N=10 \\
\text { per group }\end{array}$ & $\begin{array}{l}\mathrm{Cl}:-0.7435 \text { to }-0.3263 \\
p=0.0001\end{array}$ \\
\hline $\begin{array}{l}\text { Fmr1 KO veh vs } \\
\text { Fmr1 KO } 5 \mu \mathrm{M} \\
\text { simvastatin }\end{array}$ & Normally distributed & Sidak's post hoc & $\begin{array}{l}N=10 \\
\text { per group }\end{array}$ & $\begin{array}{l}\mathrm{Cl}:-0.8045 \text { to }-0.3873 \\
p<0.0001\end{array}$ \\
\hline
\end{tabular}

Figure $1 D$, metabolic labelling of protein synthesis with $0.1-0.5 \mu \mathrm{M}$ simvastatin/vehicle

\begin{tabular}{|c|c|c|c|c|}
\hline & Normally distributed & Two-way RM ANOVA & $\begin{array}{l}N=9 \\
\text { per group }\end{array}$ & $\begin{array}{l}\text { Treatment: } p<0.0001 \text {, } \\
\text { genotype: } \\
p=0.0068\end{array}$ \\
\hline WT veh vs Fmr1 KO veh & Normally distributed & Sidak's post hoc & $\begin{array}{l}N=9 \\
\text { per group }\end{array}$ & $\begin{array}{l}\mathrm{Cl}:-0.2483 \text { to }-0.06400 \\
p=0.0005\end{array}$ \\
\hline $\begin{array}{l}\text { WT veh vs WT } 0.3 \mu \mathrm{M} \\
\text { simvastatin }\end{array}$ & Normally distributed & Sidak's post hoc & $\begin{array}{l}N=9 \\
\text { per group }\end{array}$ & $\begin{array}{l}\mathrm{Cl}:-0.2760 \text { to }-0.07980 \\
p=0.0002\end{array}$ \\
\hline $\begin{array}{l}\text { Fmr1 KO veh vs } \\
\text { Fmr1 KO } 0.3 \mu \mathrm{M} \\
\text { simvastatin }\end{array}$ & Normally distributed & Sidak's post hoc & $\begin{array}{l}N=9 \\
\text { per group }\end{array}$ & $\begin{array}{l}\mathrm{Cl}:-0.2334 \text { to }-0.03724 \\
p=0.0035\end{array}$ \\
\hline $\begin{array}{l}\text { Fmr1 KO veh vs } \\
\text { Fmr1 KO } 0.5 \mu \mathrm{M} \\
\text { simvastatin }\end{array}$ & Normally distributed & Sidak's post hoc & $\begin{array}{l}N=9 \\
\text { per group }\end{array}$ & $\begin{array}{l}\mathrm{Cl}:-0.3121 \text { to }-0.1159 \\
p<0.0001\end{array}$ \\
\hline $\begin{array}{l}\text { WT } 0.3 \mu \mathrm{M} \text { simvastatin vs } \\
\text { Fmr1 KO } 0.3 \mu \mathrm{M} \\
\text { simvastatin }\end{array}$ & Normally distributed & Sidak's post hoc & $\begin{array}{l}N=9 \\
\text { per group }\end{array}$ & $\begin{array}{l}\mathrm{Cl}:-0.2057 \text { to }-0.02143 \\
p=0.0115\end{array}$ \\
\hline $\begin{array}{l}\text { WT } 0.5 \mu \mathrm{M} \text { simvastatin vs } \\
\text { Fmr1 KO } 0.5 \mu \mathrm{M}\end{array}$ & Normally distributed & Sidak's post hoc & $\begin{array}{l}N=9 \\
\text { per group }\end{array}$ & $\begin{array}{l}\mathrm{Cl}:-0.2210 \text { to }-0.03669 \\
p=0.0038\end{array}$ \\
\hline
\end{tabular}

Figure $2 B$, phospho/total ERK1/2 with $50 \mu \mathrm{M}$ lovastatin/vehicle Normally distributed $\quad$ Two-way RM ANOVA $\quad N=19$ per group

Genotype: $p=0.0146$

(Continued) 
Table 1. Continued

\begin{tabular}{|c|c|c|c|c|}
\hline $\begin{array}{l}\text { Figure } \\
\text { WT veh vs Fmr1 } \\
\text { KO veh }\end{array}$ & $\begin{array}{l}\text { Data structure } \\
\text { Normally distributed }\end{array}$ & $\begin{array}{l}\text { Statistical test } \\
\text { Sidak's post hoc }\end{array}$ & $\begin{array}{l}\text { Sample size } \\
N=19 \\
\text { per group }\end{array}$ & $\begin{array}{l}\text { Statistical data } \\
\mathrm{Cl}:-0.02577 \text { to } 0.1893 \\
p=0.1539\end{array}$ \\
\hline \multirow[t]{5}{*}{$\begin{array}{l}\text { Fmr1 KO veh vs } \\
\qquad \text { Fmr1 KO lovastatin }\end{array}$} & Normally distributed & Sidak's post hoc & $\begin{array}{l}N=19 \\
\text { per group }\end{array}$ & $\begin{array}{l}\mathrm{Cl}: 0.04797 \text { to } 0.2630 \\
p=0.0048\end{array}$ \\
\hline & \multicolumn{3}{|c|}{ Figure $2 C$, phospho/total ERK1/2 with $0.1-0.5 \mu \mathrm{M}$ simvastatin/vehicle } & \\
\hline & Normally distributed & Two-way RM ANOVA & $\begin{array}{l}N=11 \\
\text { per group }\end{array}$ & $\begin{array}{l}\text { Genotype: } p=0.7010 \\
\text { treatment: } p=0.8761\end{array}$ \\
\hline & Normally distributed & Two-way RM ANOVA & $\begin{array}{l}N=10 \\
\text { per group }\end{array}$ & $\begin{array}{l}\text { Genotype: } p=0.2860 \\
\text { treatment: } p=0.6206\end{array}$ \\
\hline & \multicolumn{3}{|c|}{ Figure $3 B$, AGS incidence with $3 \mathrm{mg} / \mathrm{kg}$ simvastatin } & \\
\hline $\begin{array}{l}\text { WT veh vs Fmr1 } \\
\text { KO veh }\end{array}$ & Non-normal distribution & $\begin{array}{l}\text { Two-tailed Fisher's exact } \\
\text { test }\end{array}$ & $\begin{array}{l}N=12 \\
\text { per group }\end{array}$ & $\begin{array}{l}\mathrm{Cl}: 0.002672 \text { to } 0.3437, \\
p=0.0028\end{array}$ \\
\hline
\end{tabular}

Figure 3C, AGS severity distribution scores with $3 \mathrm{mg} / \mathrm{kg}$ simvastatin

WT veh vs Fmr1 $\mathrm{KO}$ veh

Fmr1 KO veh vs Fmr1 KO

simvastatin

\section{Fmr1 KO veh vs Fmr1 KO} simvastatin

WT veh vs Fmr1 $\mathrm{KO}$ veh

WT simvastatin vs Fmr1 $\mathrm{KO}$ simvastatin

Fmr1 KO veh vs Fmr1 KO simvastatin

WT veh vs Fmr1 $\mathrm{KO}$ veh

Fmr1 KO veh vs Fmr1 KO simvastatin

Fmr1 KO veh vs Fmr1 KO simvastatin

WT veh vs Fmr1 $\mathrm{KO}$ veh

WT lovastatin vs Fmr1 KO lovastatin

$\begin{array}{llll}\text { Non-normal distribution } & \text { Mann-Whitney test } & N=12 & \text { Cl: } 0.000 \text { to } 2.000, \\ & & \text { per group } & p=0.0028 \\ \text { Non-normal distribution } & \text { Mann-Whitney test } & N=12 & \mathrm{Cl}:-1.000 \text { to } 1.000, \\ & & \text { per group } & p=0.9510\end{array}$

Figure $3 D$, AGS latency with $3 \mathrm{mg} / \mathrm{kg}$ simvastatin

\begin{tabular}{|c|c|}
\hline Normally distributed & $\begin{array}{l}\text { Unpaired two-tailed } t \\
\text { test }\end{array}$ \\
\hline
\end{tabular}

Figure $3 E$, AGS incidence with $50 \mathrm{mg} / \mathrm{kg}$ simvastatin
Non-normal distribution
Two-tailed Fisher's exact
KO veh: $n=14$ test
WT veh: $n=12$
Cl: 0.004960 to 0.5143 ,
$p=0.0053$
Non-normal distribution
Two-tailed Fisher's exact
KO simva: $n=11$
$\mathrm{Cl}: 0.006556$ to 0.7356 , test
WT simva: $n=13$
$p=0.0233$
Non-normal distribution
Two-tailed Fisher's exact test
KO veh: $n=14$
KO simva: $n=11$
$\mathrm{Cl}: 0.2988$ to 7.531 ,
$p=0.6968$

Figure 3F, AGS severity scores with $50 \mathrm{mg} / \mathrm{kg}$ simvastatin

$\begin{array}{lll}\text { Non-normal distribution } & \text { Mann-Whitney test } & \text { KO veh: } n=14 \\ & & \text { WT veh: } n=12 \\ \text { Non-normal distribution } & \text { Mann-Whitney test } & \text { KO veh: } n=14 \\ & & \text { KO simva: } n=11\end{array}$

Cl: 0.000 to 3.000 ,

$p=0.0036$

Cl: -3.000 to 0.000 ,

$p=0.2254$

Figure 3G, AGS latency with $50 \mathrm{mg} / \mathrm{kg}$ simvastatin

\begin{tabular}{|c|c|c|c|}
\hline Normally distributed & $\begin{array}{l}\text { Unpaired two-tailed } \\
t \text { test }\end{array}$ & $\begin{array}{l}\text { KO veh: } n=14 \\
\text { KO simva: } n=11\end{array}$ & $\begin{array}{l}\mathrm{Cl}:-11.41 \text { to } 8.739 \\
p=0.7794\end{array}$ \\
\hline
\end{tabular}

Figure $3 H$, AGS incidence with $100 \mathrm{mg} / \mathrm{kg}$ lovastatin

$\begin{array}{ccc}\text { Non-normal distribution } & \text { Two-tailed Fisher's exact } & \text { KO veh: } n=16 \\ & \text { test } & \text { WT veh: } n=15 \\ \text { Non-normal distribution } & \text { Two-tailed Fisher's exact } & \text { KO lova: } n=14 \\ & \text { test } & \text { WT lova: } n=17\end{array}$

Cl: 0.01126 to 0.4341 , $p=0.0032$

Cl: 0.06948 to 3.440 , $p=0.6358$

(Continued) 
Table 1. Continued

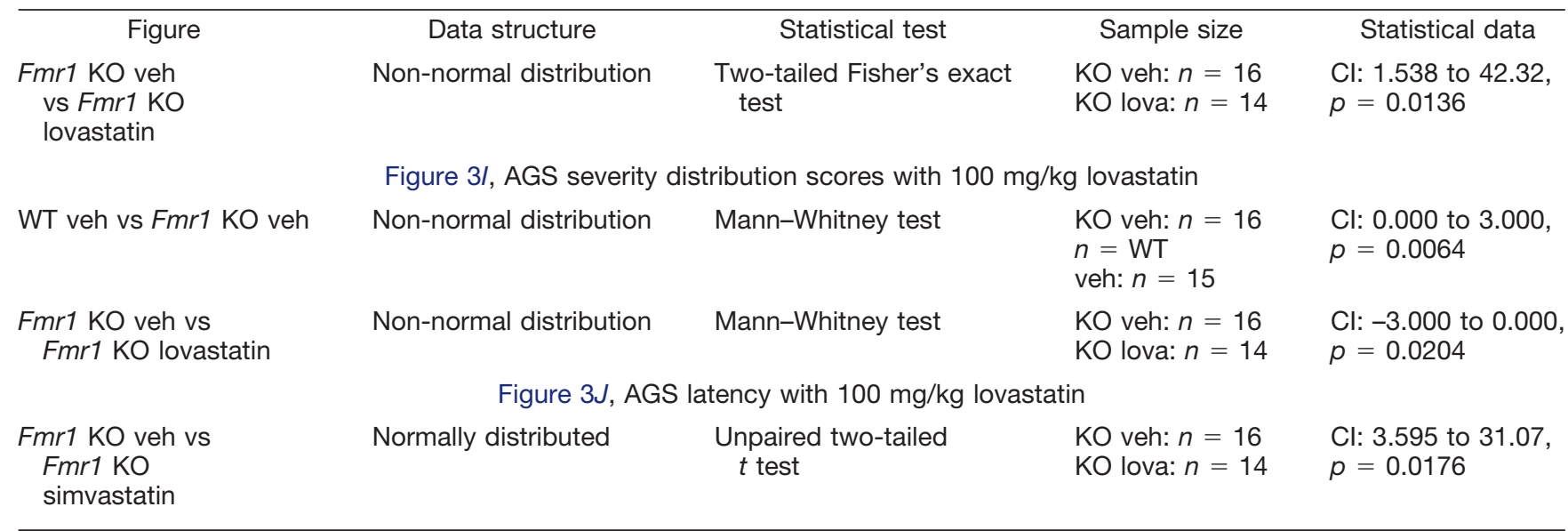

$=116.52 \pm 2.21 \%, \mathrm{KO} 0.3 \mu \mathrm{M}=129.15 \pm 3.99 \%, \mathrm{KO}$ $0.5 \mu \mathrm{M}=137.01 \pm 3.08 \%$; WT veh vs $0.3 \mu \mathrm{M} p=0.0002$, WT veh vs $0.5 \mu \mathrm{M} p<0.0001, \mathrm{KO}$ veh vs $0.3 \mu \mathrm{M} p=$ $0.0035, \mathrm{KO}$ veh vs $0.5 \mu \mathrm{M} p<0.0001 ; n=9$; Fig. $1 D$ ). These results show that unlike lovastatin, simvastatin does not correct excessive protein synthesis in the $\mathrm{Fmr1}^{-/ y}$ hippocampus.

\section{Lovastatin, but not simvastatin, reduces ERK1/2 activation}

Our metabolic labeling experiments show that $50 \mu \mathrm{M}$ lovastatin reduces protein synthesis in the $F m r 1^{-/ y}$ hippocampus by 15-20\% (Fig. 1B). Conversely, $0.5 \mu \mathrm{M}$ simvastatin causes a 15-20\% increase in protein synthesis in the $F m r 1^{-/ y}$ hippocampus (Fig. 1D). Given the opposite
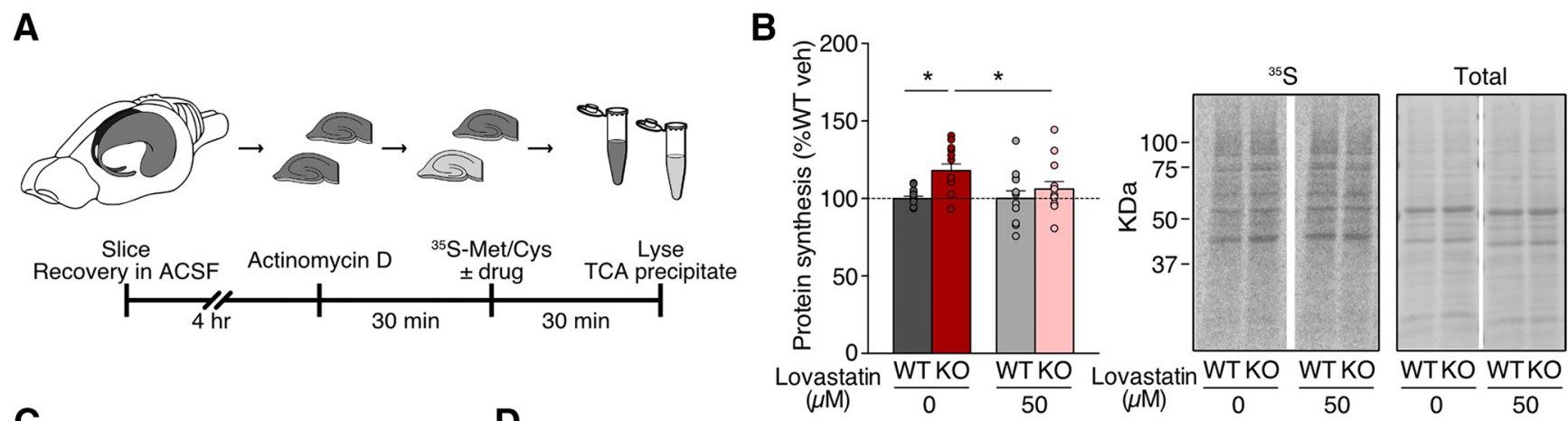

C

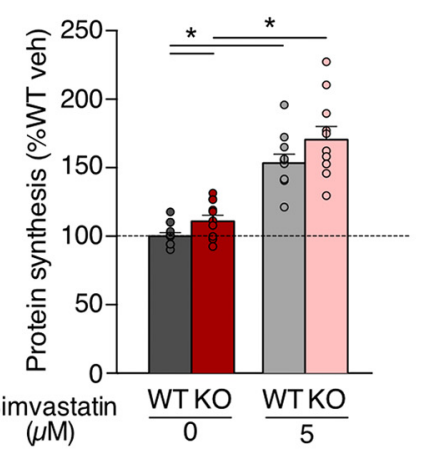

D

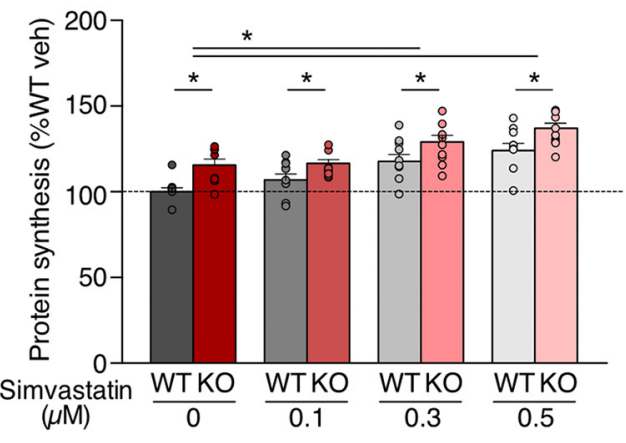

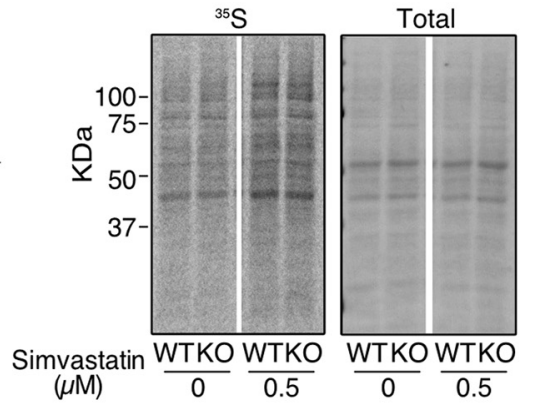

Figure 1. Simvastatin exaggerates excessive protein synthesis in the $F m r 1^{-1 /}$ hippocampus. Slices were prepared from WT and $\mathrm{Fmr}^{-/ y}$ hippocampi and incubated in vehicle, lovastatin, or simvastatin at different concentrations. A, Schematic shows time course for metabolic labeling experiments of hippocampal slices. $\boldsymbol{B}$, Lovastatin significantly decreases protein synthesis in $F m r 1^{-/ y}$ slices to WT levels (ANOVA genotype $* p=0.0106$; Sidak's WT veh vs KO veh $* p=0.0032$, KO veh vs KO lova $* p=0.0368 ; n=12$ ). $\boldsymbol{C}$, Simvastatin raises protein synthesis in both WT and $F m r 1^{-1 y}$ slices at $5 \mu \mathrm{M}$ (ANOVA treatment $* p<0.0001$, genotype $* p=0.0294$; Sidak's WT veh vs $5 \mu \mathrm{M} * p=0.0001$, KO veh vs $5 \mu \mathrm{M} * p<0.0001 ; n=10)$. $\boldsymbol{D}$, Simvastatin raises protein synthesis at $0.1-0.5 \mu \mathrm{M}$, exaggerating the excessive protein synthesis phenotype (ANOVA treatment $* p<0.0001$, genotype $* p=0.0068$; Sidak's WT veh vs $0.3 \mu \mathrm{M} * p=0.0002$, WT veh vs $0.5 \mu \mathrm{M} * p<0.0001$, KO veh vs $0.3 \mu \mathrm{M} * p=0.0035$, KO veh vs $0.5 \mu \mathrm{M} * p<0.0001$, WT veh vs KO veh $* p=0.0005$, WT $0.1 \mu \mathrm{M}$ vs KO $0.1 \mu \mathrm{M} * p=0.0406$, WT $0.3 \mu \mathrm{M}$ vs KO $0.3 \mu \mathrm{M} * p=0.0115$, WT $0.5 \mu \mathrm{M}$ vs KO $0.5 \mu \mathrm{M} * p$ $=0.0038 ; n=9$ ). Representative samples were run on SDS-PAGE gels and transferred to membranes. Example phosphorimages of ${ }^{35} \mathrm{~S}$-labeled proteins and total protein staining of the same membrane are shown. Error bars $=\mathrm{SEM}$. $N=$ littermate pairs.

Figure Contributions: Melania Muscas and Susana R. Louros performed the experiments and analyzed the data. 
A

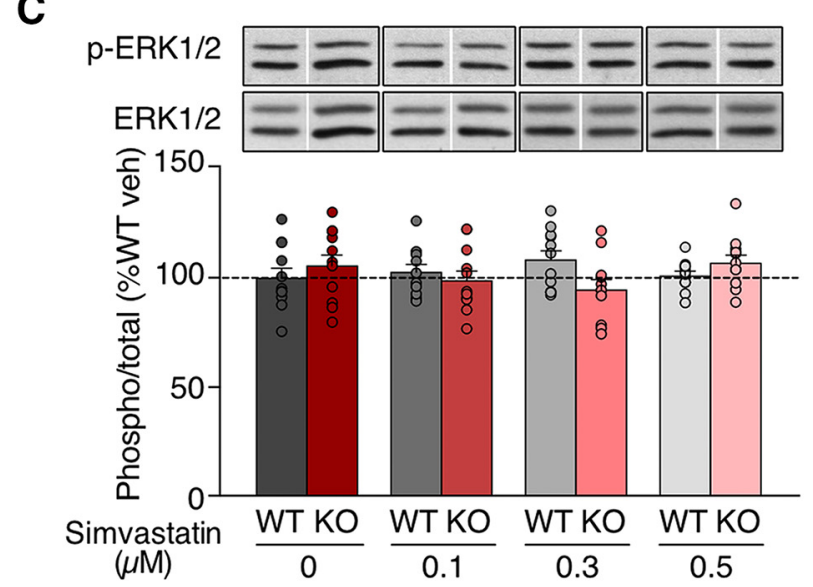

D

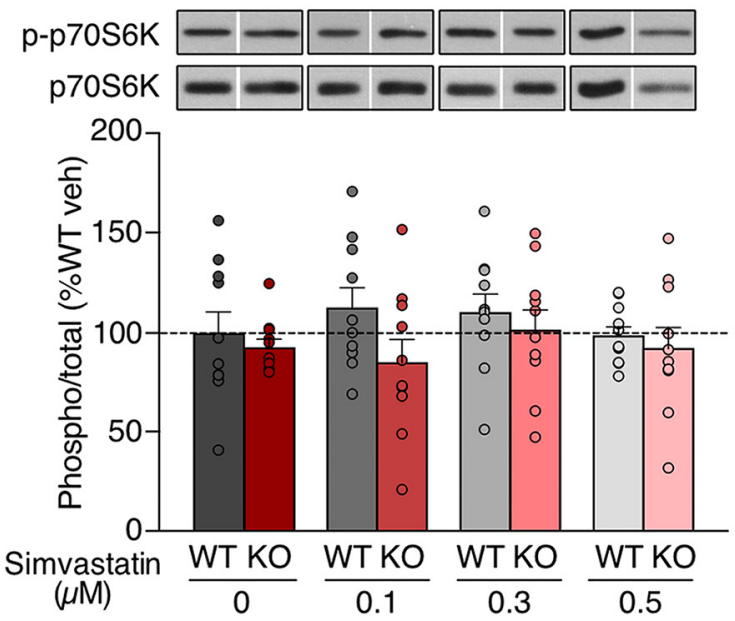

Figure 2. Simvastatin does not reduce ERK1/2 or mTORC1 activation in the $F m r 1^{-1 y}$ hippocampus. A, Diagram shows the potential impact of simvastatin on Ras-ERK1/2 and RhebmTOR-signaling pathways. B, Fmr1 $1^{-/ y}$ slices incubated with 50 $\mu \mathrm{M}$ lovastatin show a significant reduction in ERK1/2 phosphorylation (ANOVA genotype $* p=0.0146$; Sidak's KO veh vs KO lova $* p=0.0048 ; n=19)$. $\boldsymbol{C}$, Simvastatin treatment does not reduce ERK1/2 phosphorylation in $\mathrm{Fmr1}^{-/ y}$ or WT slices (ANOVA treatment $p=0.8761$, genotype $p=0.7010 ; n=11)$. $\boldsymbol{D}$, Simvastatin treatment does not reduce phosphorylation of p70S6K in WT or Fmr1 ${ }^{-/ y}$ slices (ANOVA treatment $p=0.6206$, genotype $p=0.2860 ; n=10$ ). Representative bands were cropped from original blots as indicated by blank spaces. Original blots are
Figure 2. continued

shown in Extended Data Figure 2-1. Error bars = SEM. $N=$ littermate pairs.

Figure Contributions: Melania Muscas performed the experiments and analyzed the data.

effect of lovastatin and simvastatin on protein synthesis, we wondered whether these compounds acted differently on the ERK $1 / 2$ and mTOR translation control signaling pathways (Fig. 2A). To confirm the same lovastatin treatment that reduces excess protein synthesis in the $F m r 1^{-1 y}$ also reduces ERK $1 / 2$ activation, we incubated slices in vehicle or $50 \mu \mathrm{M}$ lovastatin and performed quantitative immunoblotting for p-ERK1/2 (Fig. 2B; Extended Data Fig. 2-1). Our results confirm that $50 \mu \mathrm{M}$ lovastatin significantly reduces p-ERK1/2 in $F m r 1^{-1 y}$ slices as previously reported $($ WT veh $=100 \pm 4.32 \%$, WT lova $=99.28 \pm$ $4.42 \%, \mathrm{KO}$ veh $=91.83 \pm 4.74 \%, \mathrm{KO}$ lova $=76.28 \pm$ $3.76 \%$; KO veh vs lova $p=0.0048 ; n=19$ ).

Next, to test whether simvastatin had a differential impact on ERK1/2 signaling at the same concentration that causes a $15-20 \%$ increase in protein synthesis, we repeated our immunoblotting analysis on slices exposed to vehicle or $0.1-0.5 \mu \mathrm{M}$ simvastatin. In contrast to lovastatin, our results show that simvastatin has no significant impact on p-ERK $1 / 2$ in either WT or Fmr1 ${ }^{-1 /}$ slices at any dose tested (WT veh $=100 \pm 4.51 \%$, WT $0.1 \mu \mathrm{M}=102.87 \pm 3.42 \%$, WT $0.3 \mu \mathrm{M}=108.45 \pm$ $4.10 \%$, WT $0.5 \mu \mathrm{M}=101.01 \% \pm 2.09 \%$, KO veh $=105.63 \pm$ $4.97 \%$, KO $0.1 \mu \mathrm{M}=98.94 \pm 4.46 \%$, KO $0.3 \mu \mathrm{M}=$ $94.71 \pm 4.53 \%$, KO $0.5 \mu \mathrm{M}=106.93 \pm 3.65 \% ; n=11$; Fig. 2C; Extended Data Fig. 2-1). This suggests that simvastatin neither activates nor inhibits the ERK $1 / 2$ pathway under conditions where it increases protein synthesis.

Although our previous study with lovastatin showed no effect of lovastatin on mTOR activation as assessed by phosphorylation of p70S6K, we wondered whether simvastatin had an observable impact on this pathway. To investigate, we immunoblotted for p-p70S6K in WT and Fmr1-1/y slices treated with $0.1-0.5 \mu \mathrm{M}$ simvastatin. Our results show that p70S6K activation is unchanged in slices treated with 0.1-0.5 $\mu \mathrm{M}$ simvastatin (WT veh $=100 \pm 11.14 \%$, WT 0.1 $\mu \mathrm{M}=112.94 \pm 10.25 \%$, WT $0.3 \mu \mathrm{M}=110.66 \pm 9.47 \%$, WT $0.5 \mu \mathrm{M}=98.89 \pm 4.72 \%, \mathrm{KO}$ veh $=92.87 \pm 4.49 \%, \mathrm{KO} 0.1$ $\mu \mathrm{M}=85.37 \% \pm 11.82 \%, \mathrm{KO} 0.3 \mu \mathrm{M}=101.71 \% \pm 10.37 \%$, $\mathrm{KO} 0.5 \mu \mathrm{M}=92.53 \% \pm 10.64 \% ; n=10$; Fig. $2 D$; Extended Data Fig. 2-1). Together, these experiments show that unlike lovastatin, simvastatin does not affect the activation of ERK1/2, nor does it alter the mTORC1-p70S6K pathway.

\section{Lovastatin, but not simvastatin, corrects the AGS phenotype in the $\mathrm{Fmr1}^{-/ y}$ mouse}

Our work in vitro shows that simvastatin does not correct the ERK1/2-stimulated excess in protein synthesis in the $F m r 1^{-/ y}$ hippocampus, suggesting that it may not have the same efficacy as lovastatin in ameliorating pathologic phenotypes. To directly test this, we performed a sideby-side analysis of the effect of lovastatin versus simvastatin on the incidence of AGS in the $F m r 1^{-/ y}$ mouse. Although the AGS phenotype is seen in $\mathrm{Fmr1}^{-1 /}$ mice bred on multiple mouse background strains, a more robust 
phenotype is observed in mice bred on the FVB strain or a C57BI6/J x FVB hybrid strain (Yan et al., 2004, 2005). Therefore, we used $F m r 1^{-1 /}$ and littermate WT mice bred on a C57BI6/J x FVB hybrid strain for our AGS study. Importantly, lovastatin corrects the AGS phenotype in $\mathrm{Fmr1}^{-1 / y}$ bred on both C57BL/6J and FVB strains, suggesting the rescue is not dictated by background genetics (Osterweil et al., 2013).

To test whether simvastatin could similarly correct the AGS phenotype, we injected $F m r 1^{-1 y}$ and littermate WT mice with $3 \mathrm{mg} / \mathrm{kg}$ simvastatin as described in Materials and Methods. We used the lactone prodrug version of simvastatin administered to human patients, which is hydrolyzed into the active hydroxy acid compound by the liver (Schachter, 2005). The initial dose of simvastatin was chosen based on previous work showing $1 \mathrm{mg} / \mathrm{kg}$ simvastatin reduces epileptogenic activity and neurotoxicity in a kainic acid (KA) rat model of epilepsy (Xie et al., 2011). Additionally, according to a conversion factor of 0.081 for mouse to human dosing recommended by the Food and Drug Administration (FDA), $3 \mathrm{mg} / \mathrm{kg}$ simvastatin in mouse would be equivalent to the $20 \mathrm{mg}$ dose used in humans (Nair and Jacob, 2016).

Animals were injected with vehicle or simvastatin with the experimenter blind to genotype and treatment, and then left in a quiet environment for $1 \mathrm{~h}$ before AGS testing. A 1-h incubation time was chosen based on previous experiments using lovastatin, and on previous pharmacokinetic studies in mice and rats showing that simvastatin peaks in blood at $30 \mathrm{~min}$ to $1 \mathrm{~h}$ after administration (van de Steeg et al., 2013; Higgins et al., 2014; Xu et al., 2014), and peaks in brain $1 \mathrm{~h}$ after administration (JohnsonAnuna et al., 2005). To induce AGS, animals were transferred to a test chamber and exposed to a 2-min digitized sampling of a personal alarm passed through $50-\mathrm{W}$ speakers at a level of $>130 \mathrm{~dB}$. Seizures were recorded at increasing levels of severity as: 1, wild running (uncontrolled and undirected running); 2, clonic seizure (loss of balance with violent spasms on all limbs); and 3, tonic seizure (loss of balance with postural rigidity in limbs and tail; Fig. $3 A$ ). Latency between the onset of the AGS stimulus and seizure was also used as a metric of seizure severity and measured as the number of seconds between the start of the alarm to the first appearance of wild running.

Our results show that vehicle-treated $F m r 1^{-1 y}$ mice exhibit a significantly higher incidence of AGS versus WT littermates (WT veh $8 \%$, KO veh $75 \%, p=0.0028$ ) and a significant increase in seizure severity (WT vs KO veh $p=$ 0.0028 ). However, in contrast to lovastatin, $3 \mathrm{mg} / \mathrm{kg} \mathrm{sim}$ vastatin injection had no significant effect on the incidence of AGS in $\mathrm{Fmr1}^{-/ y}$ mice (WT veh 8\%, WT simva 9\%, KO veh $75 \%$, KO simva $75 \%$; WT vs KO simva $p=$ 0.0028 , KO veh vs simva $p=1.000$; Fig. $3 B$ ). Comparison of AGS scores showed $3 \mathrm{mg} / \mathrm{kg}$ simvastatin was similarly ineffective in reducing seizure severity (KO veh: wild running $1 / 12$, clonic $5 / 12$, tonic $3 / 12$; $K O$ simva: wild running $3 / 12$, clonic $2 / 12$, tonic $4 / 12$; KO veh vs simva $p=0.951$; Fig. $3 C$ ). Measurements of the latency to first seizure also reveal no significant effect of simvastatin treatment (KO veh $=26.33 \pm 3.80 \mathrm{~s}, \mathrm{KO}$ simva $=42.11 \pm 12.32 \mathrm{~s}, p=$ 0.239 ; Fig. $3 D$ ). These results suggest simvastatin is not effective in correcting AGS in $\mathrm{Fmr1}^{-1 /}$ mice.

Although $3 \mathrm{mg} / \mathrm{kg}$ is consistent with a simvastatin dose used in previous studies of KA-induced seizure, higher doses of up to $50 \mathrm{mg} / \mathrm{kg}$ have also been investigated with respect to neurologic phenotypes in rodents (Ramirez et al., 2011; Ling and Tejada-Simon, 2016). Indeed, intraperitoneal injection of $50 \mathrm{mg} / \mathrm{kg}$ active simvastatin $24 \mathrm{~h}$ and $30 \mathrm{~min}$ before seizure induction protects against KA-induced seizures in mice (Ramirez et al., 2011), and increases learning in a mouse model of Alzheimer's disease ( $\mathrm{Li}$ et al., 2006). To ensure that simvastatin is not effective in correcting the AGS phenotype in $\mathrm{Fmr1}^{-1 /}$ mice, we repeated our experiments using a high dose of 50 $\mathrm{mg} / \mathrm{kg}$. To remove the potential confound of prodrug metabolism, we injected active simvastatin hydroxy acid rather than inactive lactone. In a comparison group, we tested an equipotent $100 \mathrm{mg} / \mathrm{kg}$ dose of active lovastatin hydroxy acid that was previously shown to correct AGS in adult $F m r 1^{-1 y}$ FVB mice (Osterweil et al., 2013). Separate groups of $\mathrm{Fmr}^{-/ y}$ and WT littermates were injected with $50 \mathrm{mg} / \mathrm{kg}$ simvastatin or $100 \mathrm{mg} / \mathrm{kg}$ lovastatin (with corresponding vehicle) and AGS testing performed as previously.

Our results show that even at a higher dose, simvastatin does not reduce AGS incidence in Fmr1 ${ }^{-1 y}$ mice (WT veh $8 \%$, WT simva $8 \%$, KO veh $64 \%$, KO simva $55 \%$; WT vs KO veh $p=0.0053$, WT vs KO simva $p=0.0233$, KO veh vs simva $p=0.6968$; Fig. $3 E$ ). AGS severity is similarly not reduced in simvastatin-treated $F m r 1^{-1 y}$ mice as assessed by seizure score (KO veh: wild running $0 / 14$, clonic $1 / 14$, tonic $8 / 14$; $K O$ simva: wild running $0 / 11$, clonic $3 / 11$, tonic $3 / 11$; WT vs $K O$ veh $* p=0.0036, K O$ veh vs $K O$ simva $p$ $=0.2254$; Fig. $3 F$ ) or latency to seizure onset (KO veh = $27 \pm 2.95 \mathrm{~s}, \mathrm{KO}$ simva $=25.67 \pm 3.61 \mathrm{~s}, p=0.779$; Fig. $3 G)$. In contrast, $\mathrm{Fmr1}^{-1 /}$ mice injected with $100 \mathrm{mg} / \mathrm{kg}$ lovastatin showed a significant reduction in AGS versus vehicle-treated mice (WT veh 13\%, WT lova $12 \%$, KO veh $69 \%$, KO lova $21 \%$; WT vs KO veh $p=0.0032, \mathrm{KO}$ veh vs lova $p=0.0136$, WT veh vs KO lova $p=0.6513$; Fig. $3 H$ ). Additionally, AGS scoring reveals a decrease in the severity of seizures in lovastatin-treated $\mathrm{Fmr1}^{-1 / y}$ mice (KO veh: wild running $0 / 16$, clonic $5 / 16$, tonic $6 / 16$; KO lova: wild running $0 / 14$, clonic $1 / 14$, tonic $2 / 14$; WT vs $K O$ veh $* p=0.0064, \mathrm{KO}$ veh vs $\mathrm{KO}$ lova $* p=0.0204 ;$ Fig. $3 /)$, and an increase in the latency to the first seizure (KO veh $=28$ $\pm 3 \mathrm{~s}, \mathrm{KO}$ lova $=45.33 \pm 4.84 \mathrm{~s}, \mathrm{KO}$ veh vs lova $* p=$ 0.0176; Fig. 3J). Together, these results show that lovastatin reduces the incidence and severity of AGS in the $F m r 1^{-1 y}$, whereas simvastatin has no effect.

\section{Discussion}

The promising results using lovastatin in FX have led to the suggestion that simvastatin may be similarly effective. In this study, we investigated two core phenotypes in the $\mathrm{Fmr1}^{-1 / y}$ mouse model to test the prediction that simvastatin can be used in place of lovastatin. Our results show that simvastatin not only fails to correct excessive protein synthesis in the $F m r 1^{-1 y}$ hippocampus, it worsens this 
A

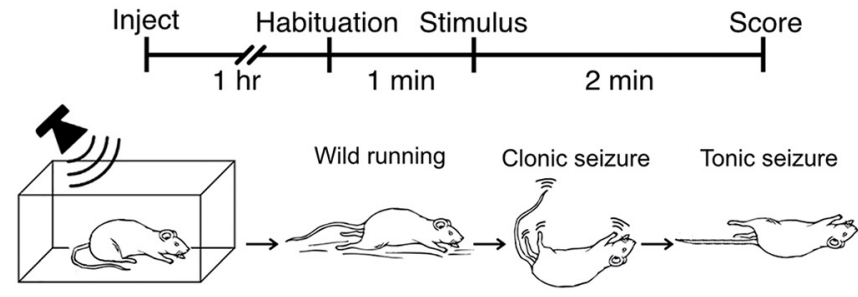

B

Simvastatin - Low dose

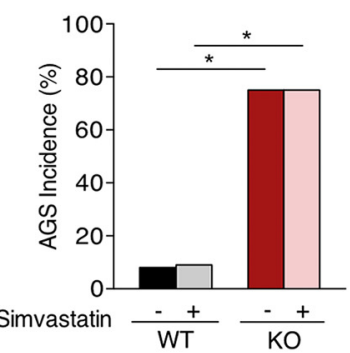

E

Simvastatin - High dose

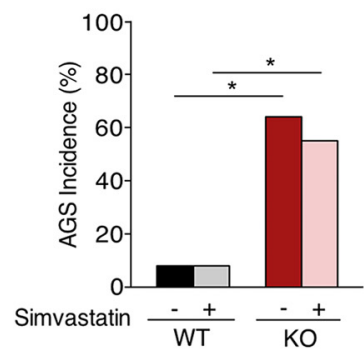

H

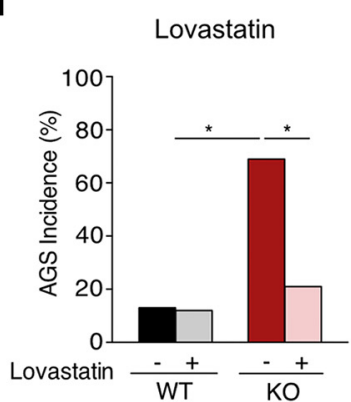

C

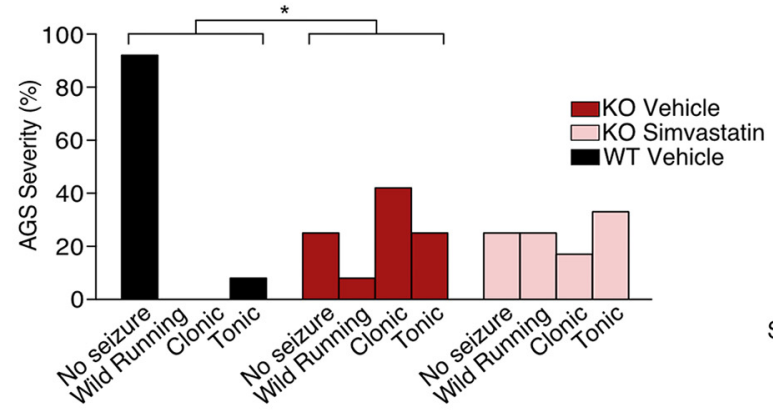

F

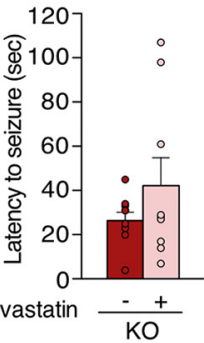

G

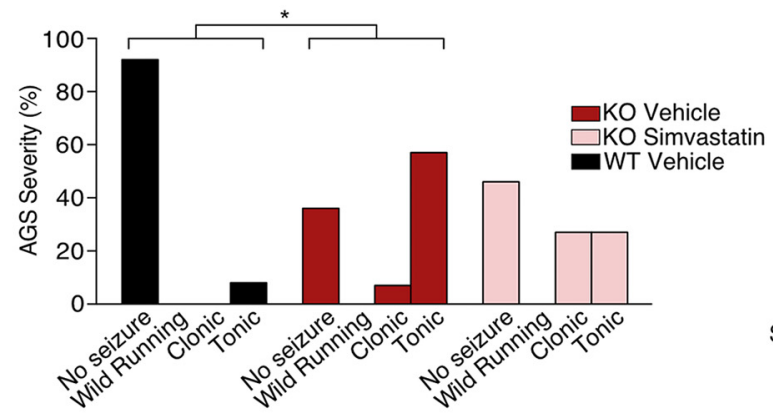

I

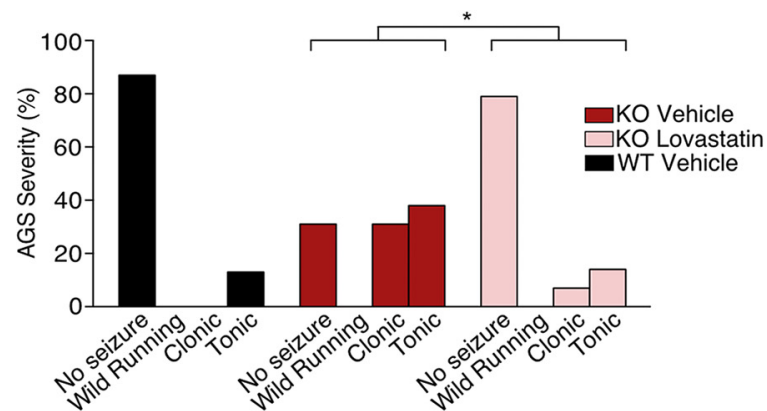

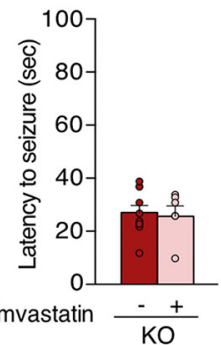

J

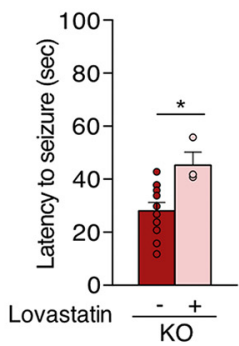

Figure 3. Simvastatin does not correct AGS in the $F m r 1^{-1 y}$ mouse. Fmr1 ${ }^{-1 y}$ and littermate WT mice were injected intraperitoneally with vehicle, simvastatin, or lovastatin and tested for AGS. A, Schematic shows the experimental timeline and scoring system for AGS testing. $\boldsymbol{B}$, Injection of $3 \mathrm{mg} / \mathrm{kg}$ simvastatin does not reduce the incidence of AGS in Fmr1 ${ }^{-/ y}$ mice (Fisher's exact test WT vs KO veh $* p=0.0028$, WT vs KO simva $* p=0.0028$, KO veh vs simva $p>0.999)$. $C$, Comparison of AGS scores also shows no reduction of seizure severity with $3 \mathrm{mg} / \mathrm{kg}$ simvastatin (Mann-Whitney WT vs KO veh $* p=0.0028$, KO veh vs KO simva $p=0.9510$ ). $\boldsymbol{D}, 3 \mathrm{mg} / \mathrm{kg}$ simvastatin does not increase latency to first seizure in $F m r 1^{-1 / y}$ mice (unpaired $t$ test $p=0.239$ ). $E, 50 \mathrm{mg} / \mathrm{kg}$ active simvastatin does not reduce AGS incidence in Fmr1 ${ }^{-1 /}$ mice (Fisher's exact test WT vs KO veh $* p=0.0053$, WT vs KO simva $* p=0.0233$, KO veh vs simva $p=0.6968)$. $\boldsymbol{F}$, AGS severity scores are not significantly reduced with $50 \mathrm{mg} / \mathrm{kg}$ simvastatin (Mann-Whitney WT vs KO veh $* p$ $=0.0036, \mathrm{KO}$ veh vs KO simva $p=0.2254)$. $\mathbf{G}$, Latency to first seizure is not significantly different between vehicle and $50 \mathrm{mg} / \mathrm{kg}$ simvastatin-treated $F m r 1^{-1 y}$ mice (unpaired $t$ test $p=0.779$ ). $\boldsymbol{H}$, Injection of $100 \mathrm{mg} / \mathrm{kg}$ lovastatin significantly reduces the incidence of AGS in Fmr1 ${ }^{-1 y}$ mice (Fisher's exact test WT vs KO veh $* p=0.0032$, WT vs KO lova $p=0.6358$, KO veh vs lova $* p=0.0136$ ). I, Lovastatin reduces severity scores of AGS in $\mathrm{Fmr1}^{-/ y}$ mice versus vehicle (Mann-Whitney WT vs KO veh $* p=0.0064$, KO veh vs 
continued

KO lova $* p=0.0204)$. $J$, Lovastatin treatment significantly increases the latency to first seizure compared to vehicle-treated $F m r 1^{-/ y}$ mice (unpaired $t$ test KO veh vs lova $* p=0.0176$ ). Error bars $=\mathrm{SEM}$.

Figure Contributions: Melania Muscas performed the experiments and analyzed the data.

phenotype (Fig. 1). We do not see a reduction of ERK1/2 activation at the concentrations of simvastatin tested (Fig. 2). Moreover, simvastatin does not reduce the incidence or severity of AGS in the $F m r 1^{-1 y}$ mouse even at a high dose of $50 \mathrm{mg} / \mathrm{kg}$ (Fig. 3). These results suggest that simvastatin should not be assumed to be an effective replacement for lovastatin with respect to correction of $\mathrm{Fmr1}^{-/ y}$ pathology.

Although we propose the beneficial effect of lovastatin stems from the inhibition of ERK1/2-driven protein synthesis, it is important to note that statins are capable of affecting several biochemical pathways. Beyond the canonical impact on cholesterol biosynthesis, statins also decrease isoprenoid intermediates including farnesyl and geranylgeranyl pyrophosphates that regulate membrane association for many proteins including the small GTPases Ras, Rho, and Rac (Schafer et al., 1989; Liao and Laufs, 2005; Nürenberg and Volmer, 2012; Ling and Tejada-Simon, 2016). The increase in protein synthesis seen with simvastatin could be linked to altered posttranslational modification of these or other proteins. Indeed, although we see no change in mTORC1-p70S6K signaling, other studies have shown an activation of the PI3 kinase pathway that could be contributing to this effect (Mans et al., 2010). However, our comparison of lovastatin and simvastatin shows that there is a clear difference in the correction of pathology in the $F \mathrm{mr}^{-/ y}$ model, suggesting that the impact on ERK1/2 is an important factor in terms of pharmacological treatment for FX.

There are many reasons why statins would be an attractive option for treating neurodevelopmental disorders such as FX. They are prescribed worldwide for the treatment of hypercholesterolemia and coronary heart disease (Istvan, 2003), and safely used for long-term treatment in children and adults (Ling and Tejada-Simon, 2016). However, our study suggests that care should be taken when considering which statin should be trialed for the treatment of FX and other disorders of excess Ras. Although the effect of different statins on cholesterol synthesis has been well documented, the differential impact on RasERK1/2 signaling is not well established. We show here that, contrary to lovastatin, simvastatin fails to inhibit the Ras-ERK1/2 pathway in the $F m r 1^{-/ y}$ hippocampus, exacerbates the already elevated protein synthesis phenotype, and does not correct the AGS phenotype. These results are significant for considering future studies with lovastatin or simvastatin in FX or other disorders of excess Ras. Indeed, clinical trials using simvastatin for the treatment of NF1 have shown little promise, while trials with lovastatin show an improvement in cognitive deficits (van der Vaart et al., 2013; Bearden et al., 2016; Payne et al., 2016). Although further studies testing a broader dose range of simvastatin on additional $\mathrm{Fmr1}^{-1 / y}$ brain phenotypes will ultimately determine the feasibility of this strat- egy for FX, our study suggests caution should be used when assuming simvastatin is a suitable substitute for lovastatin.

\section{References}

Alabama-Birmingham U, NCI 2009 (2010) A randomized placebocontrolled study of lovastatin in children with neurofibromatosis type 1 (STARS). ClinicalTrialsgov. Available at https://clinicaltrials. gov/ct2/show/NCT00853580. Accessed on March 12, 2018.

Ashley CT Jr, Wilkinson KD, Reines D, Warren ST (1993) FMR1 protein: conserved RNP family domains and selective RNA binding. Science 262:563-566.

Bear MF, Huber KM, Warren ST (2004) The mGluR theory of fragile $X$ mental retardation. Trends Neurosci 27:370-377.

Bearden CE, Hellemann GS, Rosser T, Montojo C, Jonas R, Enrique N, Pacheco L, Hussain SA, Wu JY, Ho JS, McGough JJ, Sugar CA, Silva AJ (2016) A randomized placebo-controlled lovastatin trial for neurobehavioral function in neurofibromatosis I. Ann Clin Transl Neurol 3:266-279.

Berry-Kravis E (2002) Epilepsy in fragile X syndrome. Dev Med Child Neurol 44:724-728.

Berry-Kravis EM, Lindemann L, Jonch AE, Apostol G, Bear MF, Carpenter RL, Crawley JN, Curie A, Des Portes V, Hossain F, Gasparini F, Gomez-Mancilla B, HessI D, Loth E, Scharf SH, Wang PP, Von Raison F, Hagerman R, Spooren W, Jacquemont S (2017) Drug development for neurodevelopmental disorders: lessons learned from fragile X syndrome. Nat Rev Drug Discovery 17:280299.

Busquets-Garcia A, Gomis-González M, Guegan T, Agustín-Pavón C, Pastor A, Mato S, Pérez-Samartín A, Matute C, de la Torre R, Dierssen M, Maldonado R, Ozaita A (2013) Targeting the endocannabinoid system in the treatment of fragile $X$ syndrome. Nat Med 19:603-607.

Çaku A, Pellerin D, Bouvier P, Riou E, Corbin F (2014) Effect of lovastatin on behavior in children and adults with fragile $X$ syndrome: an open-label study. Am J Med Genet A 164A:2834-2842.

Darnell JC, Van Driesche SJ, Zhang C, Hung KY, Mele A, Fraser CE, Stone EF, Chen C, Fak JJ, Chi SW, Licatalosi DD, Richter JD, Darnell RB (2011) FMRP stalls ribosomal translocation on mRNAs linked to synaptic function and autism. Cell 146:247-261.

Dölen G, Osterweil E, Rao BS, Smith GB, Auerbach BD, Chattarji S, Bear MF (2007) Correction of fragile $X$ syndrome in mice. Neuron 56:955-962.

Hagerman RJ, Berry-Kravis E, Kaufmann WE, Ono MY, Tartaglia N, Lachiewicz A, Kronk R, Delahunty C, HessI D, Visootsak J, Picker J, Gane L, Tranfaglia M (2009) Advances in the treatment of fragile X syndrome. Pediatrics 123:378-390.

Higgins JW, Bao JQ, Ke AB, Manro JR, Fallon JK, Smith PC, Zamek-Gliszczynski MJ (2014) Utility of Oatp1a/1b-knockout and OATP1B1/3-humanized mice in the study of OATP-mediated pharmacokinetics and tissue distribution: case studies with pravastatin, atorvastatin, simvastatin, and carboxydichlorofluorescein. Drug Metab Dispos 42:182-192.

Istvan E (2003) Statin inhibition of HMG-CoA reductase: a 3-dimensional view. Atheroscler Suppl 4:3-8.

Johnson-Anuna LN, Eckert GP, Keller JH, Igbavboa U, Franke C, Fechner T, Schubert-Zsilavecz M, Karas M, Müller WE, Wood WG (2005) Chronic administration of statins alters multiple gene expression patterns in mouse cerebral cortex. J Pharmacol Exp Ther 312:786-793.

Johnson-Anuna LN, Eckert GP, Franke C, Igbavboa U, Müller WE, Wood WG (2007) Simvastatin protects neurons from cytotoxicity 
by up-regulating Bcl-2 mRNA and protein. J Neurochem 101:7786.

Jones P, Kafonek S, Laurora I, Hunninghake D (1998) Comparative dose efficacy study of atorvastatin versus simvastatin, pravastatin, lovastatin, and fluvastatin in patients with hypercholesterolemia (the CURVES study). Am J Cardiol 81:582-587.

King MK, Jope RS (2013) Lithium treatment alleviates impaired cognition in a mouse model of fragile $X$ syndrome. Genes Brain Behav 12:723-731.

Krab LC, de Goede-Bolder A, Aarsen FK, Pluijm SM, Bouman MJ, van der Geest JN, Lequin M, Catsman CE, Arts WF, Kushner SA, Silva AJ, de Zeeuw Cl, Moll HA, Elgersma $Y$ (2008) Effect of simvastatin on cognitive functioning in children with neurofibromatosis type 1: a randomized controlled trial. JAMA 300:287-294.

Li L, Cao D, Kim H, Lester R, Fukuchi K (2006) Simvastatin enhances learning and memory independent of amyloid load in mice. Ann Neurol 60:729-739.

Li W, Cui Y, Kushner SA, Brown RA, Jentsch JD, Frankland PW, Cannon TD, Silva AJ (2005) The HMG-CoA reductase inhibitor lovastatin reverses the learning and attention deficits in a mouse model of neurofibromatosis type 1. Curr Biol 15:1961-1967.

Liao JK, Laufs U (2005) Pleiotropic effects of statins. Annu Rev Pharmacol Toxicol 45:89-118.

Lim JH, Lee JC, Lee YH, Choi IY, Oh YK, Kim HS, Park JS, Kim WK (2006) Simvastatin prevents oxygen and glucose deprivation/ reoxygenation-induced death of cortical neurons by reducing the production and toxicity of 4-hydroxy-2E-nonenal. J Neurochem 97:140-150.

Ling Q, Tejada-Simon MV (2016) Statins and the brain: new perspective for old drugs. Prog Neuropsychopharmacol Biol Psychiatry 66:80-86.

Lozano R, Rosero CA, Hagerman RJ (2014) Fragile X spectrum disorders. Intractable Rare Dis Res 3:134-146.

Mans RA, Chowdhury N, Cao D, McMahon LL, Li L (2010) Simvastatin enhances hippocampal long-term potentiation in C57BL/6 mice. Neuroscience 166:435-444.

Mendola CE, Backer JM (1990) Lovastatin blocks N-ras oncogeneinduced neuronal differentiation. Cell Growth Differ 1:499-502.

Michalon A, Sidorov M, Ballard TM, Ozmen L, Spooren W, Wettstein JG, Jaeschke G, Bear MF, Lindemann L (2012) Chronic pharmacological mGlu5 inhibition corrects fragile $X$ in adult mice. Neuron 74:49-56.

Musumeci SA, Bosco P, Calabrese G, Bakker C, De Sarro GB, Elia M, Ferri R, Oostra BA (2000) Audiogenic seizures susceptibility in transgenic mice with fragile $X$ syndrome. Epilepsia 41:19-23.

Nair AB, Jacob $S$ (2016) A simple practice guide for dose conversion between animals and human. J Basic Clin Pharm 7:27-31.

Neuvonen PJ, Backman JT, Niemi M (2008) Pharmacokinetic comparison of the potential over-the-counter statins simvastatin, lovastatin, fluvastatin and pravastatin. Clin Pharmacokinet 47:463474.

Nürenberg G, Volmer DA (2012) The analytical determination of isoprenoid intermediates from the mevalonate pathway. Anal Bioanal Chem 402:671-685.

Osterweil EK, Krueger DD, Reinhold K, Bear MF (2010) Hypersensitivity to mGluR5 and ERK1/2 leads to excessive protein synthesis in the hippocampus of a mouse model of fragile $X$ syndrome. $J$ Neurosci 30:15616-15627.

Osterweil EK, Chuang SC, Chubykin AA, Sidorov M, Bianchi R, Wong RK, Bear MF (2013) Lovastatin corrects excess protein synthesis and prevents epileptogenesis in a mouse model of fragile $X$ syndrome. Neuron 77:243-250.

Payne JM, Barton B, Ullrich NJ, Cantor A, Hearps SJ, Cutter G, Rosser T, Walsh KS, Gioia GA, Wolters PL, Tonsgard J, Schorry E, Viskochil D, Klesse L, Fisher M, Gutmann DH, Silva AJ, Hunter SJ, Rey-Casserly C, Cantor NL, et al. (2016) Randomized placebocontrolled study of lovastatin in children with neurofibromatosis type 1. Neurology 87:2575-2584.
Pellerin D, Caku A, Fradet M, Bouvier P, Dube J, Corbin F (2016) Lovastatin corrects ERK pathway hyperactivation in fragile $X$ syndrome: potential of platelet's signaling cascades as new outcome measures in clinical trials. Biomarkers 21:497-508.

Qin M, Kang J, Burlin TV, Jiang C, Smith CB (2005) Postadolescent changes in regional cerebral protein synthesis: an in vivo study in the FMR1 null mouse. J Neurosci 25:5087-5095.

Ramirez C, Tercero I, Pineda A, Burgos JS (2011) Simvastatin is the statin that most efficiently protects against kainate-induced excitotoxicity and memory impairment. J Alzheimers Dis 24:161-174.

Schachter M (2005) Chemical, pharmacokinetic and pharmacodynamic properties of statins: an update. Fundam Clin Pharmacol 19:117-125.

Schaefer EJ, McNamara JR, Tayler T, Daly JA, Gleason JL, Seman LJ, Ferrari A, Rubenstein JJ (2004) Comparisons of effects of statins (atorvastatin, fluvastatin, lovastatin, pravastatin, and simvastatin) on fasting and postprandial lipoproteins in patients with coronary heart disease versus control subjects. Am J Cardiol 93:31-39.

Schafer WR, Kim R, Sterne R, Thorner J, Kim SH, Rine J (1989) Genetic and pharmacological suppression of oncogenic mutations in ras genes of yeast and humans. Science 245:379-385.

Sharma A, Hoeffer CA, Takayasu Y, Miyawaki T, McBride SM, Klann E, Zukin RS (2010) Dysregulation of mTOR signaling in fragile $X$ syndrome. J Neurosci 30:694-702.

Stoppel LJ, Osterweil EK, Bear MF (2017a) The mGluR theory from mice to men. In: Fragile $X$ syndrome: from genetics to targeted treatment (Willemsen R, Kooy F, eds). London: Elsevier.

Stoppel LJ, Kazdoba TM, Schaffler MD, Preza AR, Heynen A, Crawley JN, Bear MF (2017b) R-Baclofen reverses cognitive deficits and improves social interactions in two lines of $16 \mathrm{p} 11.2$ deletion mice. Neuropsychopharmacology 43:513-524.

Tsuji A, Saheki A, Tamai I, Terasaki T (1993) Transport mechanism of 3-hydroxy-3-methylglutaryl coenzyme $A$ reductase inhibitors at the blood-brain barrier. J Pharmacol Exp Ther 267:1085-1090.

van der Vaart T, Plasschaert E, Rietman AB, Renard M, Oostenbrink $R$, Vogels $A$, de Wit MC, Descheemaeker MJ, Vergouwe $Y$, Catsman-Berrevoets CE, Legius E, Elgersma Y, Moll HA (2013) Simvastatin for cognitive deficits and behavioural problems in patients with neurofibromatosis type 1 (NF1-SIMCODA): a randomised, placebo-controlled trial. Lancet Neurol 12:1076-1083.

van de Steeg E, Kleemann R, Jansen HT, van Duyvenvoorde W, Offerman EH, Wortelboer HM, Degroot J (2013) Combined analysis of pharmacokinetic and efficacy data of preclinical studies with statins markedly improves translation of drug efficacy to human trials. J Pharmacol Exp Ther 347:635-644.

Wang X, Snape M, Klann E, Stone JG, Singh A, Petersen RB, Castellani RJ, Casadesus G, Smith MA, Zhu X (2012) Activation of the extracellular signal-regulated kinase pathway contributes to the behavioral deficit of fragile x-syndrome. J Neurochem 121: 672-679.

Xie C, Sun J, Qiao W, Lu D, Wei L, Na M, Song Y, Hou X, Lin Z (2011) Administration of simvastatin after kainic acid-induced status epilepticus restrains chronic temporal lobe epilepsy. PLoS One 6:e24966.

Xu D, Li F, Zhang M, Zhang J, Liu C, Hu MY, Zhong ZY, Jia LL, Wang DW, Wu J, Liu L, Liu XD (2014) Decreased exposure of simvastatin and simvastatin acid in a rat model of type 2 diabetes. Acta Pharmacol Sin 35:1215-1225.

Yan QJ, Asafo-Adjei PK, Arnold HM, Brown RE, Bauchwitz RP (2004) A phenotypic and molecular characterization of the fmr1-tm1Cgr fragile X mouse. Genes Brain Behav 3:337-359.

Yan QJ, Rammal M, Tranfaglia M, Bauchwitz RP (2005) Suppression of two major fragile $X$ syndrome mouse model phenotypes by the mGluR5 antagonist MPEP. Neuropharmacology 49:10531066. 